\title{
Los alimentos y bebidas ultra-procesados que ingresan a Colombia por el tratado de libre comercio ¿influirán en el peso de los colombianos?
}

\author{
Ultra-processed foods and beverages products entering \\ Colombia through international trade agreements. Will they \\ have an impact on the weight of colombians?
}

\begin{abstract}
U Itra-processed products are characterized by high contents of sugar, saturated fat and sodium. These products are entering the Colombian market through free trade agreements. O ne concern related to their consumption is the impact on weight gain and comorbidities associated with overweight and obesity, as well as changes in cultural habits and food sovereignty. Scientific evidence has documented that consumption of ultra processed products contributes to chronic disease, thus increasing health care costs of treatment and rehabilitation. We must implement public policies that protect and inform the consumer, increasing the access and the right to a healthy diet, while reducing consumption of ultra-processed products such as sugar sweetened beverages and snacks. Inter-sectorial strategies are needed to face the potential purchasing and consumption patterns changes, promoting the development of food systems that recognize local dynamics of production. Increase control over the type and quality of ultraprocessed products that are entering the country, and promote actions, along with food and nutrition education are also needed. Key Words: Free trade agreements, overweight, obesity, ultraprocessed products, food systems, food and beverages.
\end{abstract}

Jhon Bejarano-Roncancio (1) Edna Magaly Gamboa-Delgado(2) Dora Hilda Aya-Baquero (3) Diana C. Parra $(4,5)$

(1) Departamento de N utrición Humana. Facultad de Medicina. Universidad N acional de Colombia. Bogotá, Colombia (2) Fundación Cardiovascular de Colombia., Bucaramanga, Colombia

(3) Seguridad Alimentaria y Nutricional, FAO, Colombia. (4) Centre for Epidemiological Studies in Health and Nutrition, School of Public Health, U niversity of São Paulo, Sao Paulo, SP, Brazil. (5) Program in Physical Therapy, School of Medicine, Washington University in St. Louis, St. Louis, MO, USA

Dirigir la correspondencia a: Profesor thon Bejarano-Rocancio Depto. de Nutrición Humana, Fac. de Medicina U. Nacional, Colombia

Este trabajo fue recibido el 14 de Enero de 2015 y aceptado para ser publicado el 30 de Septiembre de 2015.

\section{INTRODUCCIÓN}

Las enfermedades crónicas no transmisibles son una de las principales causas de mortalidad en países desarrollados y en vía de desarrollo. Las diferencias entre las dietas consumidas por la población tienen importantes implicaciones en la morbilidad y mortalidad. Diversos estudios han demostrado la fuerte asociación entre el consumo de alimentos y bebidas ultra-procesados, y enfermedades crónicas no transmisibles (1-5). Una nueva clasificación de los alimentos ha sido propuesta por Monteiro y Cols, la cual tiene en cuenta la naturaleza, el alcance y la finalidad del procesamiento de alimentos (6). Esta clasificación divide los alimentos en tres grupos: los alimentos in natura o mínimamente procesados (grupo 1), ingredientes culinarios procesados (grupo 2), y alimentos listos para consumir, ya sean procesados (grupo 3) o ultra-procesados (grupo 4).7 Los alimentos y bebidas ultraprocesados se caracterizan por su alto contenido calórico y bajo o nulo aporte de fibra, de sustancias bioactivas y ácidos grasos poliinsaturados (8). Además, estos alimentos presentan un importante contenido de ácidos grasos saturados y trans, colesterol, azúcar y sodio (1)
Por sus características, el consumo y la compra regular de productos ultra-procesados se han visto asociado a una mayor incidencia de síndrome metabólico y obesidad $(2,8-12,16)$.

Un reciente reporte de la Organización Panamericana de la Salud13 ha identificado a los productos procesados como un importante motor de la epidemia de obesidad en la región de América Latina. El informe muestra un aumento exponencial en la venta y el consumo de este tipo de alimentos en países en los que aun predomina la tradición culinaria y gastronómica tales como Perú, Brasil y Colombia. En Colombia el crecimiento anual en la venta de alimentos y bebidas ultra procesadas durante un periodo de 13 años (2000 a 2013) fue de 90.2 kilogramos, representando un incremento del $25 \%$. Los países donde se vieron los incrementos más altos fueron Chile (59.8\%), Uruguay (146.4\%), Bolivia (129.8\%) y Perú (107\%). Estos cambios responden a políticas económicas y de mercados que incluyen tratados de libre comercio y otros tipos de acuerdo de libre inversión en la región. Se ha documentado el impacto que tienen las políticas de apertura económica y la desregulación de los mercados sobre la salud de las poblaciones en lo referente a la obesidad y las enfer- 
medades cronicas $(14,15)$.

En el marco de los sistemas alimentarios, se encuentran sistemas tradicionales y modernos que coexisten y evolucionan con la urbanización y la globalización. Las cadenas de suministro modernas traen consigo la integración vertical del almacenamiento, la distribución y el expendio al por menor, lo cual aumenta la eficiencia y reduce el costo de ciertos alimentos. Aunque estos sistemas mixtos ofrecen variedad de alimentos nutritivos, también favorecen la venta masiva de alimentos y bebidas ultra procesados que pueden contribuir al exceso de peso y enfermedades crónicas no transmisibles, si se consumen en exceso $(17,26)$.

El objetivo de este artículo es analizar el posible impacto que tendrán los distintos tratados de libre comercio (TLC) a los que se está sometiendo Colombia, sobre el consumo de alimentos y bebidas ultra-procesados, y cómo esto afectará la dieta y el peso de los colombianos, así como las posibles implicaciones sobre la soberanía alimentaria. Se tomó como referencia la evidencia publicada, algunas experiencias internacionales $(18,25,26)$ y un análisis detallado de las políticas de importación con Estados Unidos de América (USA). Finalmente, se sintetizan algunos aspectos claves para motivar acciones y mecanismos de alerta al Estado y la sociedad científica y civil.

\section{El Tratado de Libre Comercio y los alimentos y bebidas ultra-procesados.}

Los TLC son convenios entre dos o más países en los cuales se acuerdan normas que facilitan el comercio entre ellos mediante el libre intercambio de sus alimentos y servicios. En un TLC prima el intercambio comercial y deben ser respetados los derechos y principios consagrados en la Constitución o marco legal de cada Estado. Estos acuerdos se rigen por el derecho internacional público, y buscan aumentar el bienestar de la población mediante un mayor crecimiento económico y social (27) Las acciones entre ellos, son mediadas y facilitadas por los acuerdos comerciales regionales de la Organización Mundial del Comercio (OMC). Una de las categorías de alimentos que ingresarán a Colombia a través de los acuerdos de TLC con USA, Canadá y Corea del Sur, son una gran variedad y cantidad de alimentos, entre los que se encuentran productos ultra-procesados. Estos alimentos y bebidas tienen una composición no balanceada en cuanto a su distribución de macro-nutrientes, en especial por su contenido de sodio, azúcar, y grasas saturadas. Adicionalmente, cuentan con otras sustancias químicas como aditivos, que le proporcionan color, sabor artificial y textura, y de esta manera favorecen sus características para la compra y consumo. Sin embargo existe evidencia del efecto nocivo que muchos de estos productos tienen sobre la salud incluyendo algunos reconocidos como potenciales carcinógenos (28-32).

La transición nutricional en América Latina, se ha visto caracterizada por un aumento en el consumo de alimentos y bebidas ricos en azúcares refinados de alta densidad energética, así como en sal y grasa, pero que a su vez tienen escaso contenido de micronutrientes $(33,34)$ En su gran mayoría estos cambios en las dietas han sido el resultado del procesamiento industrial de los alimentos.

Alimentos que empiezan a ingresar a Colombia por el TLC.

Diversos productos alimentarios primarios y otros manufacturados por la industria, están ingresando al país por el TLC, los cuales están amparados en el Decreto 993 del 15 de mayo de 2012 (35), que aprobó el acuerdo de promoción comercial y acuerdo bilateral entre Colombia y USA. Entre los alimentos que hacen parte de la lista de bienes de consumo, se encuentran las carnes de ovino, caprino y porcino, el poIlo, pescados frescos refrigerados, congelados, ahumados, en conserva y harinas a partir de éstos, y diferentes crustáceos; aceites y grasas como la mantequilla, cereales como el arroz, el maíz y la cebada, leguminosas como la soya, azúcares como la glucosa y la fructosa, y alimentos endulzados como bombones, caramelos, chicles y demás confites.

De igual manera para la industria alimentaria, ingresan químicos como el tripolifosfato de sodio (emulsificante), ácido benzoico (conservante), glutamato monosódico (potenciador de sabor), azúcares químicamente puros, vitaminas y colorantes. Este tipo de alimentos y bebidas agudizan más la transición alimentaria y los cambios en la canasta familiar, dado que se estimula directa e indirectamente el consumo de los productos ultra-procesados, más cuando ya se tiene un comportamiento de compra y consumo. De acuerdo con la Encuesta Nacional de Situación Nutricional en Colombia realizada en 2010 , el $36,6 \%$ de los colombianos entre 5 y 64 años consume diariamente golosinas o dulces, $22,1 \%$ gaseosas o refrescos y $15,2 \%$ alimentos de paquete (36). Estos datos muestran que incluso antes de la entrada en vigor de los TLC, ya se han modificado los hábitos alimentarios y responden a una mala selección de alimentos industrializados, situación que empeora con la entrada de nuevos alimentos que serán altamente publicitados y de fácil alcance económico.

Claramente el consumidor colombiano está sometido a una variedad de alimentos que competirán con los elaborados en el mercado interno. Una de las preocupaciones que surge es que no hubo una selectividad de esos alimentos desde la composición nutricional ni desde sus implicaciones en el consumo. De igual manera se sigue motivando la utilización de sustancias químicas en los suelos y en los cultivos de frutas y hortalizas, lo que implica una producción primaria de alimentos con tecnologías poco amigables al medio ambiente. Este tema requiere ser analizado desde la inocuidad alimentaria y en toda la cadena productiva, sumado al impacto nocivo sobre el ambiente y sobre la salud que tienen la mayoría de estos químicos.

En cuanto a las políticas de importación y según cifras del DANE, en el primer semestre del 2013 Colombia compró US $\$ 238$ millones en alimentos y bebidas, destinados al consumo de los hogares, y US\$874,8 millones en alimentos procesados (37).

\section{Consumo de alimentos y bebidas ultra procesados y su impacto en la salud}

La producción y el consumo de bebidas y alimentos procesados industrialmente han incrementado de manera paralela el sobrepeso, la obesidad y otras enfermedades crónicas no transmisibles relacionadas (2). Los alimentos y bebidas ultra procesados tienen propiedades que pueden conducir a su consumo en exceso a través de mecanismos como su alta palatabilidad, grandes tamaños de porción, largos períodos de conservación y fácil transporte, lo cual favorece su consumo en cualquier momento $(38,39)$.

En Colombia, un estudio basado en información sobre la compra de alimentos según la Encuesta Nacional de Ingresos y Gastos (ENIG), encontró que la mitad de la energía comprada en los hogares $(54,6 \%)$ provino de alimentos no procesados o mínimamente procesados, $28,4 \%$ a partir de ingredientes culinarios elaborados y $16,8 \%$ se derivó de alimentos y bebidas 
ultra procesados. La dieta de los colombianos, en general, superó los límites superiores recomendados por la OMS en cuanto a grasa, azúcar y sodio. Además, el consumo de aceites de origen vegetal y de azúcar de mesa fue mayor en Colombia que los reportados en países como Brasil y Canadá (Aceites: 13,4\% Colombia vs 11,3\% Brasil y 2,3\% Canadá) (Azúcar: $10,9 \%$ Colombia vs $12,4 \%$ Brasil y $2,3 \%$ Canadá) (40).

Por otra parte, resultados de un estudio reciente en Brasil señalan que la contribución promedio de alimentos y bebidas procesados y ultra procesados al total de energía dietaria disponible estuvo en un rango entre $15.4 \%$ a $39.4 \%$. Este mismo estudio encontró una asociación positiva entre la disponibilidad de alimentos ultra procesados en el hogar y el Índice de Masa Corporal (IMC), con la prevalencia de exceso de peso y la obesidad.2 Un estudio reciente de Chile documentó que el gasto del hogar dedicado a productos ultra procesados pasó de $42 \%$ a $52 \%$ en un periodo de tres décadas (41).

Uno de los componentes más sobresalientes de los productos ultra-procesados son los ácidos grasos trans (AGT), obtenidos a partir de aceites vegetales parcialmente hidrogenados. Estudios epidemiológicos han mostrado una fuerte asociación positiva entre consumo de AGT y riesgo de enfermedad coronaria (42). Un meta-análisis de estudios de cohortes prospectivas señaló que un incremento de 2 \% en la energía proveniente de AGT estaba asociado con el incremento de la incidencia de enfermedad coronaria en $25 \%$. También existe evidencia sobre los efectos de los AGT en la resistencia a la insulina y el incremento del riesgo de diabetes mellitus (43).

De manera similar, otro componente importante de estos alimentos es el alto contenido de azúcar de las bebidas endulzadas como las gaseosas, tés, jugos de frutas industrializados, bebidas energéticas, y otras bebidas carbonatadas y no carbonatadas.44,45 El consumo de bebidas azucaradas se ha incrementado en las últimas dos décadas $(46,47)$.

Evidencia actual sugiere que la alta ingesta de bebidas azucaradas se ha visto asociada con exceso de consumo calórico, el cual lleva a ganancia de peso. Se ha encontrado que quienes consumen una o más bebidas por día, tienen el doble de riesgo de presentar diabetes mellitus y aumentan su riesgo de enfermedad coronaria en un $23 \%$, comparado con quienes consumen una o menos bebidas azucaradas por mes $(48,49)$. También se ha encontrado asociación entre el consumo de este tipo de bebidas y riesgo de obesidad e hipertensión, 50 y más recientemente con un elevado riesgo de mortalidad y discapacidad por varias causas incluyendo diabetes mellitus (133,000 muertes/año), enfermedad cardiovascular $(45,000$ muertes/año) y cánceres (6,450 muertes/año). El 70\% de estas muertes ocurren en países de medianos ingresos y México tiene la mortalidad más elevada por consumo de bebidas azucaradas con 405 muertes por un millón de adultos o el $12.1 \%$ de la mortalidad (51).

\section{Planteando soluciones y cambios a futuro}

Además del impacto negativo que tiene el consumo de alimentos y bebidas ultra procesados en la salud de las poblaciones, existen otros impactos tales como la pérdida de tradiciones agrícolas y culturales asociados con la producción y consumo de alimentos regionales, los cambios en la composición de la canasta familiar tradicional, y el menor compromiso del Estado en la producción agrícola y la soberanía alimentaria.

En Colombia, la tasa del producto interno bruto nacional dedicado al sector agrícola ha ido disminuyendo progresivamente desde el 2009, al igual que el porcentaje de empleo en el sector agrícola. De igual manera existen factores agravantes y que pueden aumentar la vulnerabilidad de los consumidores ante la llegada de alimentos y bebidas ultra procesados a través de los TLC. Estos factores incluyen: la falta de claridad del costo-beneficio en la compra, consumo y aprovechamiento biológico de los productos ultra-procesados, la falta de herramientas y conocimiento para identificar y entender el etiquetado de estos alimentos (tipo de ingredientes y su contenido nutricional) por parte del consumidor, sumado a la gran confusión y dificultad con la que se transmite esta información por parte de la industria, la limitación y/o competencia de los alimentos colombianos clasificados como mínima o moderadamente procesados, la dificultad en los controles de calidad del ingreso de productos ultra-procesados, así como el seguimiento en su comercialización durante todas las fases de la cadena productiva.

El potencial de los sistemas alimentarios para reducir la malnutrición va más allá del papel fundamental de la agricultura en la producción de alimentos y generación de ingresos. Se requieren también mecanismos de regulación del mercado y la comercialización, ligados a la protección de los consumidores, así como intervenciones en salud, saneamiento, educación y otros sectores. Es muy importante y necesario implementar programas de educación alimentaria y nutricional a nivel de las escuelas y a nivel de la familia que demuestren la facilidad y el costo efectividad de adoptar dietas basadas en alimentos con la preparación de platos caseros. Este tipo de alimentación en Colombia aún sigue siendo más barato, más accesible y más saludable, ya que el consumidor tiene mayor control sobre el contenido de azúcar, aceite y sal que decide consumir. En Colombia, la mayoría de la energía consumida proviene de preparaciones culinarias $(54,6 \%),(40)$ pero es muy probable que esto tienda a disminuir con la entrada, el fácil acceso de compra a precios muy competitivos, y el mercadeo y la publicidad agresiva de nuevos productos ultra-procesados.

Una transición a dietas sostenibles,(7) entendidas como dietas con bajo impacto ambiental que contribuyen a la seguridad alimentaria y nutricional y a la vida sana de las generaciones presentes y futuras, tendrá implicaciones significativas para los productores, la industria alimentaria, los consumidores, el uso del suelo y las normas comerciales. Estos retos requieren mecanismos de gobernanza inclusivos y basados en la evidencia que puedan abordar el análisis del sistema alimentario y su impacto sobre la naturaleza y la salud de las personas. Para el caso del exceso de peso y las ENT asociadas a la dieta, las intervenciones desde la perspectiva del complejo biomédico, están suficientemente soportadas e implican grandes asignaciones presupuestales para la salud. Sin embargo, si las intervenciones se centraran también en los determinantes sociales y económicos, incluyendo el fortalecimiento de sistemas alimentarios eficientes y dietas sostenibles, el impacto en el largo plazo sería mayor.

Es claro que muchos de los acuerdos internacionales de mercado ya establecidos serán muy difíciles de modificar, sin embargo, sí se deben prender las alarmas de la comunidad médica y de los ciudadanos para estar mejor informados y para que exijan su derecho a una alimentación saludable que priorice y apoye los alimentos y las culturas tradicionales de Colombia. El énfasis debe estar en apoyar el sector agrícola colombiano así como la agricultura local y a menor escala en el marco de sistemas de abastecimiento local de alimentos tal como ha sido recomendado por Olivier de Schutter y por agencias internacionales reconocidas como la organización de las Naciones Unidas para la Alimentación y la Agricultura - 
FAO y la Organización Mundial y Panamericanas de la Salud (OMS/OPS) $(50,51)$.

Agradecimientos: Diana C. Parra recibe apoyo a través de una beca de post doctorado con la Fundação de Amparo à Pesquisa do Estado de São Paulo (FAPESP), São Paulo, Brasil (Número de proceso 2013/08129-2) y de Washington University in St. Louis, Escuela de Medicina, programa de Terapia Física. Edna Gamboa recibe apoyo de Contrato 734-2013 Colciencias, Plan de Fortalecimiento.

\section{RESUMEN}

Los alimentos ultra-procesados se caracterizan por su alto contenido de azúcares, grasas saturadas y sodio. Estos alimentos están ingresando a Colombia por los Tratados de Libre Comercio (TLC). Una preocupación de su consumo es el impacto en la ganancia de peso progresiva y las comorbilidades asociadas al sobrepeso y la obesidad, así como el cambio de hábitos culturales y la soberanía alimentaria. La evidencia científica destaca que estos alimentos pueden generar enfermedades crónicas lo que incrementa los costos sanitarios debido al tratamiento y rehabilitación. Es necesario formular políticas públicas que protejan y orienten al consumidor, facilitando el acceso y el derecho a una alimentación saludable y mitigando el consumo de productos ultra-procesados como las bebidas azucaradas y los empaquetados. Se requieren estrategias y actores intersectoriales para hacer frente a los posibles cambios de compra y consumo, promover el desarrollo de sistemas alimentarios que reconozcan las dinámicas locales de producción. Realizar vigilancia y control sobre los productos ultra-procesados que ingresan al país y promover acciones de educación alimentaria y nutricional.

Palabras clave: Tratados de libre comercio, sobrepeso, obesidad, alimentos ultra-procesados, sistemas alimentarios, alimentos y bebidas.

\section{BIBLIOGRAFIA}

1. Moubarac, J. C. et al. Consumption of ultra-processed foods and likely impact on human health. Evidence from Canada. Public Health Nutr. 2012;16: 2240-48.

2. Canella, D. S. et al. Ultra-processed food products and obesity in Brazilian households (2008-2009). PloS one. 2014; 9, e92752, doi:10.1371/journal.pone.0092752.

3. Martins, A. P., Levy, R. B., Claro, R. M., Moubarac, J. C. \& Monteiro, C. A. Increased contribution of ultra-processed food products in the Brazilian diet (1987-2009). Rev Saude Publica. 2013;47: 656-65.

4. Da Costa LM, Bortoletto AP, Silva D, Galastri L, Bertazzi $\mathrm{R}$, Moreira $\mathrm{R}$ et al . Impact of ultra-processed foods on micronutrient content in the Brazilian diet. Rev. Saúde Pública. 2015; 49: 1-8.

5. Singh G, Micha R, Khatibzadeh S, Lim S, Ezzati M, Mozaffarian D. Estimated Global, Regional, and National Disease Burdens Related to Sugar-Sweetened Beverage Consumption in 2010. J Am Heart Assoc. 2015. http:// goo.gl/wdS2Om

6. Monteiro, C. A., Levy, R. B., Claro, R. M., Castro, I. R. \& Cannon, G. A new classification of foods based on the extent and purpose of their processing. Cad Saude Publica. 2010; 26: 2039-49.

7. Monteiro CA, Cannon G, Moubarac JC, Martins AP, Martins CA, Garzillo J, Canella DS, Baraldi LG, Barciotte $M$, Louzada $M L$, Levy RB, Claro RM, Jaime PC. Dietary guidelines to nourish humanity and the planet in the twenty-first century. A blueprint from Brazil. Public Health Nutr. 2015; 24:1-12.

http://www.ncbi.nlm.nih.gov/pubmed/26205679

8. Louzada MLC, Martins AP, Canella D et al. (2015) Impact of ultra-processed foods on micronutrient content in the Brazilian diet. Rev Saude Publica (In the Press).

9. Moubarac, J. C., Parra, D. C., Cannon, G. \& Monteiro, C. A. Food Classification Systems Based on Food Processing: Significance and Implications for Policies and Actions: A Systematic Literature Review and Assessment. Curr Obes Rep. 2014; 3: 256-72.

10. Mirmiran, P., Bahadoran, Z., Delshad, H. \& Azizi, F. Effects of energy-dense nutrient-poor snacks on the incidence of metabolic syndrome: a prospective approach in Tehran Lipid and Glucose Study. Nutrition. 2014;30:538-43.

11. Louzada ML, Baraldi LG, Steele EM, Martins AP, Canella DS, Moubarac JC, Levy RB, Cannon G, Afshin A, Imamura F, Mozaffarian D, Monteiro CA. Consumption of ultraprocessed foods and obesity in Brazilian adolescents and adults. Prev Med. 2015 29;81:9-15.

12. Juul F1, Hemmingsson E. Trends in consumption of ultraprocessed foods and obesity in Sweden between 1960 and 2010. Public Health Nutr. 2015; 25:1-12.

13. OMS/OPS. Alimentos y bebidas ultraprocesados en América Latina: tendencias, efecto sobre la obesidad e implicaciones para las políticas públicas. Washington D.C., 2015. http://goo.gl/ZZz2TH

14. De Vogli, R., Kouvonen, A. \& Gimeno, D. The influence of market deregulation on fast food consumption and body mass index: a cross-national time series analysis. Bulletin of the World Health Organization. 2014;92:99-107.

15. Gostin, L. O. Healthy living needs global governance. Nature. 2014; 511: 147-9.

16. Freudenberg, N. Lethal But Legal: Corporations, Consumption, and Protecting Public Health. Oxford University Press, 2004.

17. FAO. The State of Food and Agriculture - 2013. food systems for better. Food and Agriculture Organization of the United Nations, Rome, 2013.

18. Hawkes, $C$. The role of foreign direct investment in the nutrition transition. Public Health Nutr. 2005; 8: 357-65.

19. Thow, A. M. Trade liberalisation and the nutrition transition: mapping the pathways for public health nutritionists. Public Health Nutr. 2009;12:2150-8.

20. Thow, A. M., Jan, S., Leeder, S. \& Swinburn, B. The effect of fiscal policy on diet, obesity and chronic disease: a systematic review. Bull World Health Organization. 2010; 88: 609-14.

21. Thow, A. M. \& McGrady, B. Protecting policy space for public health nutrition in an era of international investment agreements. Bull World Health Organization. 2014; 92: 139-45.

22. Thow, A. M. \& Priyadarshi, S. Aid for Trade: an opportunity to increase fruit and vegetable supply. Bull World Health Organization. 2013;91:57-63.

23. Thow, A. M. et al. Taxing soft drinks in the Pacific: implementation lessons for improving health. Health Promotion Internat. 2011;26:55-64.

24. Thow, A. M. et al. The role of policy in improving diets: experiences from the Pacific Obesity Prevention in Communities food policy project. Obesity Rev. 2011;12 (Suppl 2): $68-74$

25. Arroyo, P., Loria, A. \& Mendez, O. Changes in the household calorie supply during the 1994 economic crisis in Mexico and its implications on the obesity epidemic. 
Nutr Rev. 2004; 62: S163-8.

26. Hawkes, C. Uneven dietary development: linking the policies and processes of globalization with the nutrition transition, obesity and diet-related chronic diseases. Globalization Health. 2006; 2: 4.

27. ICA. El ABC de la admisibilidad sanitaria para los productos agropecuarios colombianos en los mercados internacionales. Instituto Colombiano Agropecuario, Bogota, 2013.

28. Jacobs DR, Pereira MA, Meyer KA et al. Fiber from whole grains, but not refined grains, is inversely associated with all-cause mortality in older women: the lowa women's health study. J Am Coll Nutr; (2000) 19, 3 Suppl., S326S30.

29. Miller ER 3rd, Pastor-Barriuso R, Dalal D et al. Metaanalysis: high-dosage vitamin E supplementation may increase all-cause mortality. Ann Intern Med. 2005; 142, 37-46.

30. Omenn GS, Goodman GE, Thornquist MD et al. Effects of a combination of $\beta$ carotene and vitamin $A$ on lung cancer and cardiovascular disease. N Engl J Med. 1996; 334, 1150-5.

31. Klein EA, Thompson IM Jr, Tangen CM et al. Vitamin E and the risk of prostate cancer: the selenium and vitamin E cancer prevention trial (SELECT). JAMA (2011) 306, 1549-56.

32. Cohen D \& Farley TA (2008) Eating as an automatic behavior. Prev Chronic Dis. 2008; 5(1): A23.

33. Barría RM1, Amigo H. Nutrition transition: a review of Latin American profile. Arch Latinoam Nutr. 2006 ;56(1):3-11.

34. Mallarino, C., Gomez, L. F., Gonzalez-Zapata, L., Cadena, Y. \& Parra, D. C. Advertising of ultra-processed foods and beverages: children as a vulnerable population. Rev Saude Publica. 2013; 47: 1006-10.

35. República de Colombia. Decreto 993 de 2012. Por medio del cual se promulga el "Acuerdo de promoción comercial entre la República de Colombia y los Estados Unidos de América", sus "Cartas Adjuntas" y sus "Entendimientos". Diario Oficial No. 48.431, mayo de 2012. http://goo.gl/ p3vnso

36. ICBF. Encuesta Nacional de la Situacion Nutricional ENSIN 2010. Instituto Colombiano de Bienestar Familiar, Bogota, Colombia, 2010.

37. Agencia de noticias UN. El 21,5\% de los hogares colombianos consume alimentos ultraprocesados. Disponible en: http://goo.gl/sOl6UU
38. Monteiro, C. A., Gomes, F. S. \& Cannon, G. The snack attack. Am J Public Health. 2010; 100: 975-81.

39. Ludwig, D. S. Technology, diet, and the burden of chronic disease. JAMA. 2011;305: 1352-3.

40. Arango CM, Mejía C, Molina CF, Moubarac JC, Monteiro CA. Calidad de la dieta y su relación con el consumo de alimentos ultra-procesados: una comparación entre CoIombia, Brasil y Canadá. Consultado [13 de Septiembre 2015]. Disponible en: http://goo.gl/5RzOhh

41. Crovetto $M$, Uauy R. Changes in processed food expenditure in the population of Metropolitan Santiago in the last twenty years. Rev Med Chil. 2012 Mar;140(3):305-12

42. Iqbal, M. P. Trans fatty acids - A risk factor for cardiovascular disease. Pakistan J Med Sci. 2014;30: 194-7.

43. Odegaard, A. O. \& Pereira, M. A. Trans fatty acids, insulin resistance, and type 2 diabetes. Nutr Rev. 2006; 64: 36472.

44. Poti JM, Mendez MA, Ng SW, Popkin BM. Is the degree of food processing and convenience linked with the nutritional quality of foods purchased by US households?. Am J Clin Nutr. 2015;101(6):1251-62.

45. CDC. Does drinking beverages with added sugars increase the risk of overweight? Research to Practices Series 3 (2006).

46. Fung, T. T. et al. Sweetened beverage consumption and risk of coronary heart disease in women. Am J Clin Nutr. 2009; 89:1037-42.

47. O'Connor L, Imamura F, Lentjes MA, Khaw KT, Wareham NJ, Forouhi NG. Prospective associations and population impact of sweet beverage intake and type 2 diabetes, and effects of substitutions with alternative beverages. Diabetologia. 2015;58(7):1474-83.

48. Wang, Y. C., Coxson, P., Shen, Y. M., Goldman, L. \& Bibbins-Domingo, K. A penny-per-ounce tax on sugarsweetened beverages would cut health and cost burdens of diabetes. Health Affairs. 2012;31:199-207.

49. Schulze, M. B. et al. Sugar-sweetened beverages, weight gain, and incidence of type 2 diabetes in young and middle-aged women. JAMA. 2004;292:927-34.

50. Chen, L. et al. Reducing consumption of sugar-sweetened beverages is associated with reduced blood pressure: a prospective study among United States adults. Circulation. 2010;121:2398-406.

51. De Schutter, O. Consejo de Derechos Humanos 25 período de sesiones. Informe final: El potencial transformador del derecho a la alimentación. United Nations, Geneva, 2014. 